\title{
GAME THEORY PROBLEMS BY AN ALTERNATIVE SIMPLEX METHOD
}

\author{
Kirtiwant P. Ghadle ${ }^{1}$, Tanaji S. Pawar ${ }^{2}$

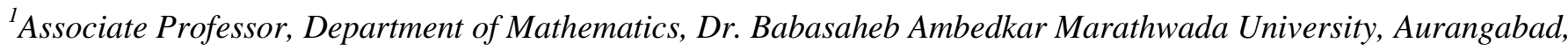 \\ Maharashtra, India \\ ${ }^{2}$ Research Student, Department of Mathematics, Dr. Babasaheb Ambedkar Marathwada University, Aurangabad, \\ Maharashtra, India
}

\begin{abstract}
In this paper, an alternative method for the solution of game problems is introduced. This method is easy to solve game problem which does not have a saddle point. It is powerful method to reduce number of iterations and save valuable time.
\end{abstract}

Keywords: Linear programming problem, Optimal solution, Alternative simplex method, and Game problem.

\section{INTRODUCTION}

Today's life is a full of struggle and competitions. A great variety of competitive situations is commonly seen. What should be the bid to win a big government job in the pace of competition from several jobs? Game must be thought of, in abroad sense, not as a kind of sport but as competitive situation, a kind of conflict in which somebody must win and somebody must lose.

John Von Neumann suggestion is to solve the game theory problems on the maximum losses. Dantzig [1] suggestion is to choose that entering vector corresponding to which $z_{j}-c_{j}$ is most negative. Khobragade et al. [2, 3, 4] suggestion is to choose that entering vector corresponding to which $\frac{\left(z_{j}-c_{j}\right) \theta_{j}}{c_{j}}$ is most negative.

In this paper, an attempt has been made to solve linear programming problem (LPP) by new method which is an alternative for simplex method. This method is different from Khobragade et al. Method.

\section{SOLUTION OF $M \quad x \quad N$ RECTANGULAR GAME PROBLEM}

By fundamental theorem of rectangular games, if mixed strategies are allowed, there always exists a value of game. (i.e. $\underline{V}=\bar{V}=V$ ).

Let the two person zero sum game be defined as follows:

Player A $\left[\begin{array}{ccc}a_{11} & \cdots & a_{1 n} \\ \vdots & \ddots & \vdots \\ a_{m 1} & \cdots & a_{m n}\end{array}\right]$

Let $p_{1}, p_{2}, \ldots, p_{m}$ and $q_{1}, q_{2}, \ldots, q_{n}$ be the probabilities of two players $\mathrm{A}$ and $\mathrm{B}$, to select their pure strategies. i.e. $S_{A}=\left(p_{1}, p_{2}, \ldots, p_{m}\right)$ and $S_{B}=\left(q_{1}, q_{2}, \ldots, q_{n}\right)$.
Then $\quad p_{1}+p_{2}+p_{3}+\ldots+p_{m}=1$

and $q_{1}+q_{2}+q_{3}+\ldots+q_{n}=1$,

Where $p_{i} \geq 0$ and $q_{j} \geq 0$ for all $i, j$.

Let the game can be defined by LPP as given below:

For player A: Minimize $X=x_{1}+x_{2}+\cdots+x_{m}$ or $=\frac{1}{V}$

Subject to: $a_{11} x_{1}+a_{21} x_{2}+\cdots+a_{m 1} x_{m} \geq 1$

$a_{12} x_{1}+a_{22} x_{2}+\cdots+a_{m 2} x_{m} \geq 1$

$a_{1 n} x_{1}+a_{2 n} x_{2}+\cdots+a_{m n} x_{m} \geq 1$

$x_{1}, x_{2}, \ldots, x_{m} \geq 0$.

For Player B: Maximize $Y=y_{1}+y_{2}+\cdots+y_{n}$ or $=\frac{1}{V}$

Subject to: $a_{11} y_{1}+a_{12} y_{2}+\cdots+a_{1 n} y_{m} \leq 1$

$a_{21} y_{1}+a_{22} y_{2}+\cdots+a_{2 n} y_{m} \leq 1$

$a_{m 1} y_{1}+a_{m 2} y_{2}+\cdots+a_{m n} y_{m} \leq 1$ $x_{1}, x_{2}, \ldots, x_{m} \geq 0$.

To find the optimal solution of the above LPP, it has been observed that the player B's problem is exactly the dual of the player A's problem. The optimal solution of one problem will automatically give the optimal solution to the other. The player B's problem can be solved by an alternative simplex method while player A's problem can be solved by an alternative dual simplex method [7]. 


\section{SOLVED PROBLEMS}

\section{1: Problem 1}

Solve the following game by linear programming technique:

Player B

Player $A\left(\begin{array}{ccc}1 & -1 & -1 \\ -1 & -1 & 3 \\ -1 & 2 & -1\end{array}\right)$.

Solution: The given game has $V=-1, \bar{V}=1$. It does not possess the saddle point and the value of game $-1<\mathrm{V}<1$. Adding a suitable constant $\mathrm{k}=1$ to all the elements of payoff of matrix.

\section{Player B}

Player A $\left(\begin{array}{lll}2 & 0 & 0 \\ 0 & 0 & 4 \\ 0 & 3 & 0\end{array}\right)$

Let the strategies of two players be:

$S_{A}=\left(p_{1}, p_{2}, p_{3}\right)$ and $S_{B}=\left(q_{1}, q_{2}, q_{3}\right)$, where $p_{1}+p_{2}+$ $p_{3}=1, q_{1}+q_{2}+q_{3}=1$.

Then, the linear programming problem can be written as:

For player A: Minimize $X=x_{1}+x_{2}+x_{3}$ or $=\frac{1}{V}$

$$
\begin{gathered}
\text { Subject to: } 2 x_{1} \geq 1 \\
3 x_{3} \geq 1 \\
4 x_{2} \geq 1 . \\
x_{1}, x_{2}, x_{3} \geq 0 .
\end{gathered}
$$

For Player B:

$$
\begin{gathered}
\text { Maximize } Y=y_{1}+y_{2}+y_{3} \text { or }=\frac{1}{V} \\
\text { Subject to: } 2 y_{1} \leq 1 \\
4 y_{3} \leq 1 \\
3 y_{2} \leq 1 \\
y_{1}, y_{2}, y_{3} \geq 0 .
\end{gathered}
$$

LPP is in standard form for player B:

$$
\begin{gathered}
\text { Maximize } Y=y_{1}+y_{2}+y_{3} \text { or }=\frac{1}{V} \\
\text { Subject to: } 2 y_{1}+s_{1}=1 \\
4 y_{3}+s_{2}=1 \\
3 y_{2}+s_{3}=1 . \\
y_{1}, y_{2}, y_{3}, s_{1}, s_{2}, s_{3} \geq 0 .
\end{gathered}
$$

Where $s_{1}, s_{2}, s_{3}$ are slack variables.

\section{Simplex Table:}

\begin{tabular}{|lll|lll|lll|l|}
\hline$C_{B}$ & BVS & $X_{B}$ & $y_{1}$ & $y_{2}$ & $y_{3}$ & $S_{1}$ & $S_{2}$ & $S_{3}$ & Ratio \\
\hline 0 & $S_{1}$ & 1 & 2 & 0 & 0 & 1 & 0 & 0 & - \\
0 & $S_{2}$ & 1 & 0 & 0 & $\underline{4}$ & 0 & 1 & 0 & $1 / 4 \rightarrow$ \\
0 & $S_{3}$ & 1 & 0 & 3 & 0 & 0 & 0 & 1 & - \\
\hline 0 & $S_{1}$ & 1 & 2 & 0 & 0 & 1 & 0 & 0 & - \\
1 & $y_{3}$ & $1 / 4$ & 0 & 0 & 1 & 0 & $1 / 4$ & 0 & - \\
0 & $S_{3}$ & 1 & 0 & $\mathbf{3}$ & 0 & 0 & 0 & 1 & $1 / 3 \rightarrow$ \\
\hline 0 & $S_{1}$ & 1 & $\underline{\mathbf{2}}$ & 0 & 0 & 1 & 0 & 0 & $1 / 2 \rightarrow$ \\
1 & $y_{3}$ & $1 / 4$ & 0 & 0 & 1 & 0 & $1 / 4$ & 0 & -
\end{tabular}

\begin{tabular}{|lll|lll|lll|l|}
1 & $y_{2}$ & $1 / 3$ & 0 & 1 & 0 & 0 & 0 & $1 / 3$ & - \\
\hline 1 & $y_{1}$ & $1 / 2$ & 1 & 0 & 0 & $1 / 2$ & 0 & 0 & \\
1 & $y_{3}$ & $1 / 4$ & 0 & 1 & 0 & 0 & $1 / 4$ & 0 & \\
1 & $y_{2}$ & $1 / 3$ & 0 & 0 & 1 & 0 & 0 & $1 / 3$ & \\
\hline
\end{tabular}

Since all rows and column are ignored, hence an optimum solution has been reached. Therefore optimum solution is:

$$
y_{1}=\frac{1}{2}, y_{2}=\frac{1}{3}, y_{3}=\frac{1}{4} . \text { Max. } Y=\frac{13}{12} \text {. }
$$

Thus, the optimal strategies for player B are:

$$
q_{1}=\frac{y_{1}}{Y}=\frac{\frac{1}{2}}{\frac{13}{12}}=\frac{6}{13}
$$

$$
q_{2}=\frac{y_{2}}{Y}=\frac{\frac{1}{3}}{\frac{12}{13}}=\frac{4}{13}
$$

$$
\begin{gathered}
q_{3}=\frac{y_{3}}{Y}=\frac{1 / 4}{12 / 13}=\frac{3}{13} \\
\text { and } V=\frac{1}{Y}-k=\frac{12}{13}-1=-\frac{1}{13} .
\end{gathered}
$$

The optimal strategies for player A are obtained from final table of the above problem. This is given by duality rules:

$$
X=Y=\frac{13}{12}
$$

$$
\begin{aligned}
& x_{1}=\left[\begin{array}{lll}
1 & 1 & 1
\end{array}\right]\left[\begin{array}{c}
1 / 2 \\
0 \\
0
\end{array}\right]=\frac{1}{2} \\
& x_{2}=\left[\begin{array}{lll}
1 & 1 & 1
\end{array}\right]\left[\begin{array}{c}
0 \\
1 / 4 \\
0
\end{array}\right]=\frac{1}{4}
\end{aligned}
$$

$$
x_{3}=\left[\begin{array}{lll}
1 & 1 & 1
\end{array}\right]\left[\begin{array}{c}
0 \\
0 \\
1 / 3
\end{array}\right]=\frac{1}{3} \text {. }
$$

Hence,

$$
\begin{gathered}
p_{1}=\frac{x_{1}}{X}=\frac{\frac{1}{2}}{\frac{13}{12}}=\frac{6}{13}, p_{2}=\frac{x_{2}}{X}=\frac{\frac{1}{4}}{\frac{13}{12}}=\frac{3}{13} \\
p_{3}=\frac{x_{3}}{X}=\frac{1 / 3}{13 / 12}=\frac{4}{13} \\
\text { and } V=\frac{12}{13}-1=\frac{-1}{13} .
\end{gathered}
$$




\section{2: Problem 2:}

Two companies $\mathrm{P}$ and $\mathrm{Q}$ are competing for the same product. Their different strategies are given in the following payoff matrix:

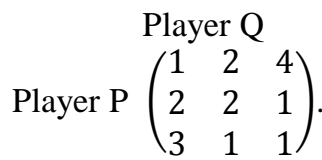

Use linear programming to determine the best strategies for both the companies.

Solution: The given game has $\underline{V}=1, \bar{V}=2$. It does not possess the saddle point and the value of game $1<\mathrm{V}<2$. Here, the payoff matrix is already non-negative.

Let the strategies of two players be:

$S_{A}=\left(p_{1}, p_{2}, p_{3}\right)$ and $S_{B}=\left(q_{1}, q_{2}, q_{3}\right)$, where $p_{1}+p_{2}+$ $p_{3}=1, q_{1}+q_{2}+q_{3}=1$.

Then, the linear programming problem can be written as:

For player A:

$$
\begin{gathered}
\text { Minimize } X=x_{1}+x_{2}+x_{3} \text { or }=\frac{1}{V} \\
\text { Subject to: } x_{1}+2 x_{2}+3 x_{3} \geq 1 \\
2 x_{1}+2 x_{2}+x_{3} \geq 1 \\
4 x_{1}+x_{2}+x_{3} \geq 1 . \\
x_{1}, x_{2}, x_{3} \geq 0 .
\end{gathered}
$$

For Player B:

$$
\begin{aligned}
& \text { Maximize } Y=y_{1}+y_{2}+y_{3} \text { or }=\frac{1}{V} \\
& \text { Subject to: } y_{1}+2 y_{2}+4 y_{3} \leq 1 \\
& 2 y_{1}+2 y_{2}+y_{3} \leq 1 \\
& 3 y_{1}+y_{2}+y_{3} \leq 1 \text {. } \\
& y_{1}, y_{2}, y_{3} \geq 0 \text {. }
\end{aligned}
$$

LPP is in standard form for player B:

$$
\begin{aligned}
& \text { Maximize } Y=y_{1}+y_{2}+y_{3} \text { or }=\frac{1}{V} \\
& \text { Subject to: } y_{1}+2 y_{2}+4 y_{3}+s_{1}=1 \\
& 2 y_{1}+2 y_{2}+y_{3}+s_{2}=1 \\
& 3 y_{1}+y_{2}+y_{3}+s_{3}=1 . \\
& y_{1}, y_{2}, y_{3}, s_{1}, s_{2}, s_{3} \geq 0 .
\end{aligned}
$$

Where $s_{1}, s_{2}, s_{3}$ are slack variables.

\section{Simplex Table:}

\begin{tabular}{|lll|lll|lll|l|}
\hline$C_{B}$ & BVS & $X_{B}$ & $y_{1}$ & $y_{2}$ & $y_{3}$ & $s_{1}$ & $s_{2}$ & $S_{3}$ & Ratio \\
\hline 0 & $S_{1}$ & 1 & 1 & 2 & $\mathbf{4}$ & 1 & 0 & 0 & $1 / 4 \rightarrow$ \\
0 & $S_{2}$ & 1 & 2 & 2 & 1 & 0 & 1 & 0 & 1 \\
0 & $S_{3}$ & 1 & 3 & 1 & 1 & 0 & 0 & 1 & 1 \\
\hline 1 & $y_{3}$ & $1 / 4$ & $1 / 4$ & $1 / 2$ & 1 & $1 / 2$ & 0 & 0 & \\
0 & $S_{2}$ & $3 / 4$ & $7 / 4$ & $3 / 2$ & 0 & $-1 / 4$ & 1 & 0 & $3 / 7$ \\
0 & $S_{3}$ & $3 / 4$ & $\mathbf{1 1 / 4}$ & $1 / 2$ & 0 & $-1 / 4$ & 0 & 1 & $3 / 11 \rightarrow$ \\
\hline 1 & $y_{3}$ & $2 / 11$ & 0 & $5 / 11$ & 1 & $3 / 11$ & 0 & - & \\
& & & & & & & & $1 / 11$ &
\end{tabular}

\begin{tabular}{|lll|lll|lll|l|}
0 & $S_{2}$ & $3 / 11$ & 0 & $\underline{\mathbf{3 3} / 11}$ & 0 & - & 1 & - & $3 / 13 \rightarrow$ \\
1 & $y_{1}$ & $3 / 11$ & 1 & $2 / 11$ & 0 & - & 0 & $4 / 11$ & \\
\hline 1 & $y_{3}$ & $1 / 13$ & 0 & 0 & 1 & $4 / 13$ & $-5 / 13$ & $2 / 13$ & \\
1 & $y_{2}$ & $3 / 13$ & 0 & 1 & 0 & - & $11 / 13$ & - & \\
& & & & & & $1 / 13$ & & $7 / 13$ & \\
1 & $y_{1}$ & $3 / 13$ & 1 & 0 & 0 & - & $-2 / 13$ & $6 / 13$ & \\
\hline
\end{tabular}

Since all rows and column are ignored, hence an optimum solution has been reached. Therefore optimum solution is:

$$
y_{1}=\frac{3}{13}, y_{2}=\frac{3}{13}, y_{3}=\frac{1}{13} . \operatorname{Max} . Y=\frac{7}{13}
$$

Thus, the optimal strategies for player B are:

$$
\begin{gathered}
q_{1}=\frac{y_{1}}{Y}=\frac{\frac{3}{13}}{\frac{7}{13}}=\frac{3}{7} \\
q_{2}=\frac{y_{2}}{Y}=\frac{\frac{3}{13}}{\frac{7}{13}}=\frac{3}{7} \\
q_{3}=\frac{y_{3}}{Y}=\frac{1 / 13}{7 / 13}=\frac{1}{7} \\
\text { and } V=\frac{1}{Y}-k=\frac{13}{7}-0=\frac{13}{7} .
\end{gathered}
$$

The optimal strategies for player A are obtained from final table of the above problem. This is given by duality rules:

$$
\begin{gathered}
X=Y=\frac{7}{13} \\
x_{1}=\left[\begin{array}{lll}
1 & 1 & 1
\end{array}\right]\left[\begin{array}{c}
4 / 13 \\
-1 / 13 \\
-1 / 13
\end{array}\right]=\frac{2}{13}, \\
x_{2}=\left[\begin{array}{lll}
1 & 1 & 1
\end{array}\right]\left[\begin{array}{c}
-5 / 13 \\
11 / 13 \\
-2 / 13
\end{array}\right]=\frac{4}{13} \\
x_{3}=\left[\begin{array}{lll}
1 & 1 & 1
\end{array}\right]\left[\begin{array}{c}
2 / 13 \\
-7 / 13 \\
6 / 13
\end{array}\right]=\frac{1}{13} .
\end{gathered}
$$

Hence,

$$
\begin{gathered}
p_{1}=\frac{x_{1}}{X}=\frac{\frac{2}{13}}{\frac{7}{13}}=\frac{2}{7}, p_{2}=\frac{x_{2}}{X}=\frac{\frac{4}{13}}{\frac{7}{13}}=\frac{4}{7}, \\
p_{3}=\frac{x_{3}}{X}=\frac{1 / 13}{7 / 13}=\frac{1}{7} \\
\text { and } V=\frac{13}{7}-0=\frac{13}{7} .
\end{gathered}
$$




\section{3: Problem 3:}

Solve the following game by linear programming technique:

\section{Player B}

Player $A\left(\begin{array}{ccc}3 & -1 & -3 \\ -3 & 3 & -1 \\ -4 & -3 & 3\end{array}\right)$.

Solution: The given game has $\underline{V}=-3, \bar{V}=3$. It does not possess the saddle point and the value of game $-3<\mathrm{V}<3$. To make this payoff matrix as positive, adding a suitable constant $k=5$ to all the elements of payoff of matrix.

Player B
Player A $\left(\begin{array}{lll}8 & 4 & 2 \\ 2 & 8 & 4 \\ 1 & 2 & 8\end{array}\right)$

Let the strategies of two players be:

$S_{A}=\left(p_{1}, p_{2}, p_{3}\right)$ and $S_{B}=\left(q_{1}, q_{2}, q_{3}\right)$, where $p_{1}+p_{2}+$ $p_{3}=1, q_{1}+q_{2}+q_{3}=1$.

Then, the linear programming problem can be written as:

For player A: Minimize $X=x_{1}+x_{2}+x_{3}$ or $=\frac{1}{V}$

$$
\begin{gathered}
\text { Subject to: } 8 x_{1}+2 x_{2}+x_{3} \geq 1 \\
4 x_{1}+8 x_{2}+2 x_{3} \geq 1 \\
2 x_{1}+4 x_{2}+8 x_{3} \geq 1 . \\
x_{1}, x_{2}, x_{3} \geq 0
\end{gathered}
$$

For Player B:

$$
\begin{gathered}
\text { Maximize } Y=y_{1}+y_{2}+y_{3} \text { or }=\frac{1}{V} \\
\text { Subject to: } 8 y_{1}+4 y_{2}+3 y_{3} \leq 1 \\
2 y_{1}+8 y_{2}+4 y_{3} \leq 1 \\
y_{1}+2 y_{2}+8 y_{3} \leq 1 . \\
y_{1}, y_{2}, y_{3} \geq 0 .
\end{gathered}
$$

LPP is in standard form for player B:

$$
\begin{array}{r}
\text { Maximize } Y=y_{1}+y_{2}+y_{3} \\
\text { Subject to: } 8 y_{1}+4 y_{2}+3 y_{3}+s_{1}=1 \\
2 y_{1}+8 y_{2}+4 y_{3}+s_{2}=1 \\
y_{1}+2 y_{2}+8 y_{3}+s_{3}=1 . \\
y_{1}, y_{2}, y_{3}, s_{1}, s_{2}, s_{3} \geq 0 .
\end{array}
$$

where $s_{1}, s_{2}, s_{3}$ are slack variables.

\section{Simplex Table:}

\begin{tabular}{|lll|lll|lll|l|}
\hline$C_{B}$ & $\mathrm{~B}$ & $X_{B}$ & $y_{1}$ & $y_{2}$ & $y_{3}$ & $S_{1}$ & $S_{2}$ & $s_{3}$ & Ratio \\
& $\mathrm{V}$ & & & & & & & & \\
\hline 0 & $S_{1}$ & 1 & $\underline{\mathbf{8}}$ & 4 & 2 & 1 & 0 & 0 & $1 / 8 \rightarrow$ \\
0 & $S_{2}$ & 1 & 2 & 8 & 4 & 0 & 1 & 0 & $1 / 2$ \\
0 & $S_{3}$ & 1 & 1 & 2 & 8 & 0 & 0 & 1 & 1 \\
\hline 1 & $y_{1}$ & $1 / 8$ & 1 & $1 / 2$ & $1 / 4$ & $1 / 8$ & 0 & 0 & \\
0 & $S_{2}$ & $3 / 4$ & 0 & 7 & $7 / 2$ & $-1 / 4$ & 1 & 0 & $3 / 14$ \\
0 & $S_{3}$ & $7 / 8$ & 0 & $3 / 2$ & $\mathbf{3 1 / 4}$ & $-1 / 8$ & 0 & 1 & $7 / 62 \rightarrow$ \\
\hline 1 & $y_{1}$ & $3 / 31$ & 1 & $14 / 31$ & 0 & $4 / 31$ & 0 & $-1 / 31$ & \\
0 & $S_{2}$ & $11 / 31$ & 0 & $\underline{\mathbf{1 9 6 / 3 1}}$ & 0 & $-6 / 31$ & 1 & - & $11 / 196$
\end{tabular}

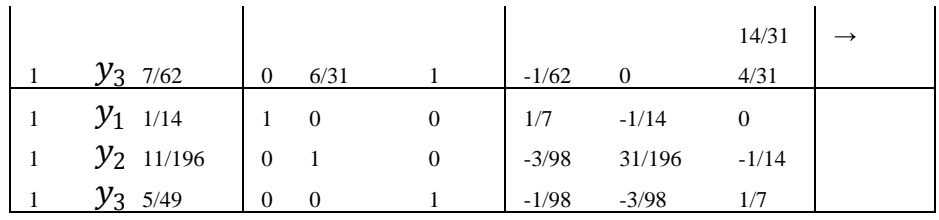

Since all rows and column are ignored, hence an optimum solution has been reached. Therefore optimum solution is:

$$
y_{1}=\frac{1}{14}, y_{2}=\frac{11}{196}, y_{3}=\frac{5}{49} . \text { Max. } Y=\frac{45}{196} \text {. }
$$

Thus, the optimal strategies for player B are:

$$
\begin{gathered}
q_{1}=\frac{y_{1}}{Y}=\frac{\frac{1}{14}}{\frac{45}{196}}=\frac{14}{45}, \\
q_{2}=\frac{y_{2}}{Y}=\frac{\frac{11}{196}}{\frac{45}{196}}=\frac{11}{45}, \\
q_{3}=\frac{y_{3}}{Y}=\frac{5 / 49}{45 / 196}=\frac{20}{45} \\
\text { and } V=\frac{1}{Y}-k=\frac{196}{45}-5=-\frac{29}{45} .
\end{gathered}
$$

The optimal strategies for player A are obtained from final table of the above problem. This is given by duality rules:

$$
\begin{gathered}
X=Y=\frac{45}{196}, \\
x_{1}=\left[\begin{array}{lll}
1 & 1 & 1
\end{array}\right]\left[\begin{array}{c}
1 / 7 \\
-3 / 98 \\
-1 / 98
\end{array}\right]=\frac{5}{49} \\
x_{2}=\left[\begin{array}{lll}
1 & 1 & 1
\end{array}\right]\left[\begin{array}{c}
-1 / 14 \\
31 / 196 \\
-3 / 98
\end{array}\right]=\frac{11}{196} \\
x_{3}=\left[\begin{array}{lll}
1 & 1 & 1
\end{array}\right]\left[\begin{array}{c}
0 \\
-1 / 14 \\
1 / 7
\end{array}\right]=\frac{1}{14} .
\end{gathered}
$$

Hence,

$$
\begin{gathered}
p_{1}=\frac{x_{1}}{X}=\frac{\frac{5}{49}}{\frac{45}{196}}=\frac{20}{45}, p_{2}=\frac{x_{2}}{X}=\frac{\frac{11}{196}}{\frac{45}{196}}=\frac{11}{45} \\
p_{3}=\frac{x_{3}}{X}=\frac{1 / 14}{45 / 196}=\frac{14}{45} \\
\text { and } V=\frac{196}{45}-5=-\frac{29}{45} .
\end{gathered}
$$




\section{4: Problem 4:}

Two companies $\mathrm{P}$ and $\mathrm{Q}$ are competing for the same product. Their different strategies are given in the following payoff matrix:

Player $\mathrm{Q}$
Player $\mathrm{P}\left(\begin{array}{ccc}-1 & 0 & 2 \\ 0 & 0 & -1 \\ 1 & -1 & -1\end{array}\right)$.

Use linear programming to determine the best strategies for both the companies.

Solution: The given game has $\underline{V}=-1, \bar{V}=0$. It does not possess the saddle point and the value of game $-1<\mathrm{V}<0$. To make this payoff matrix as positive, adding a suitable constant $k=2$ to all the elements of payoff of matrix.

Player A $\left(\begin{array}{lll}\begin{array}{l}1 \\ 2\end{array} & 2 & 4 \\ 2 & 2 & 1 \\ 3 & 1 & 1\end{array}\right)$

Let the strategies of two players be:

$S_{A}=\left(p_{1}, p_{2}, p_{3}\right)$ and $S_{B}=\left(q_{1}, q_{2}, q_{3}\right)$, where $p_{1}+p_{2}+$ $p_{3}=1, q_{1}+q_{2}+q_{3}=1$.

Then, the linear programming problem can be written as:

For player A: Minimize $X=x_{1}+x_{2}+x_{3}$ or $=\frac{1}{V}$

Subject to: $x_{1}+2 x_{2}+3 x_{3} \geq 1$

$$
\begin{gathered}
2 x_{1}+2 x_{2}+x_{3} \geq 1 \\
4 x_{1}+x_{2}+x_{3} \geq 1 . \\
x_{1}, x_{2}, x_{3} \geq 0 .
\end{gathered}
$$

Min. $Z=-\operatorname{Max} .(-Z),-Z=Z^{*}$

Max. $Z^{*}=-x_{1}-x_{2}-x_{3}$

Subject to: $-x_{1}-2 x_{2}-3 x_{3} \leq-1$

$-2 x_{1}-2 x_{2}-x_{3} \leq-1$

$-4 x_{1}-x_{2}-x_{3} \leq-1$.

$x_{1}, x_{2}, x_{3} \geq 0$.

LPP is in standard form for player A:

Max. $Z^{*}=x_{1}-x_{2}-x_{3}$

Subject to: $-x_{1}-2 x_{2}-3 x_{3}+s_{1}=-1$

$$
\begin{aligned}
& -2 x_{1}-2 x_{2}-x_{3}+s_{2}=-1 \\
& -4 x_{1}-x_{2}-x_{3}+s_{3}=-1 \\
& x_{1}, x_{2}, x_{3}, s_{1}, s_{2}, s_{3} \geq 0 .
\end{aligned}
$$

\begin{tabular}{|c|c|c|c|c|c|c|c|c|c|}
\hline$C_{B}$ & BVS & $X_{B}$ & $x_{1}$ & $x_{2}$ & $x_{3}$ & $s_{1}$ & $S_{2}$ & $s_{3}$ & Ratio \\
\hline 0 & $s_{1}$ & -1 & -1 & -2 & -3 & 1 & 0 & 0 & 1 \\
\hline 0 & $S_{2}$ & -1 & -2 & -2 & -1 & 0 & 1 & 0 & $1 / 2$ \\
\hline 0 & $S_{3}$ & -1 & -4 & -1 & -1 & 0 & 0 & 1 & $1 / 4 \rightarrow$ \\
\hline 0 & $s_{1}$ & $-3 / 4$ & 0 & $-7 / 4$ & $\begin{array}{l}= \\
11 / 4\end{array}$ & 1 & 0 & $-1 / 4$ & \\
\hline
\end{tabular}

where $s_{1}, s_{2}, s_{3}$ are slack variables.

\section{Simplex Table:}

\begin{tabular}{|lll|lll|lll|l|}
0 & $S_{2}$ & $-1 / 2$ & 0 & $-3 / 2$ & $-1 / 2$ & 0 & 0 & $-1 / 2$ & \\
-1 & $x_{1}$ & $1 / 4$ & 1 & $1 / 4$ & $1 / 4$ & 0 & 1 & $-1 / 4$ & \\
\hline-1 & $x_{3}$ & $3 / 11$ & 0 & $7 / 11$ & 1 & $-4 / 11$ & 0 & $1 / 11$ & \\
0 & $S_{2}$ & $-\underline{-4 / 11}$ & 0 & $=$ & 0 & $-2 / 11$ & 1 & $-5 / 11$ & \\
& & & & $\mathbf{1 3 / 1 1}$ & & & & & \\
-1 & $x_{1}$ & $2 / 11$ & $\mathbf{1}$ & $1 / 11$ & 0 & $1 / 11$ & 0 & $-3 / 11$ & \\
\hline-1 & $x_{3}$ & $1 / 13$ & 0 & 0 & 1 & $-6 / 13$ & $7 / 13$ & $-2 / 13$ & \\
-1 & $x_{2}$ & $4 / 13$ & 0 & 1 & 0 & $2 / 13$ & - & $5 / 13$ & \\
& & & & & & & $11 / 13$ & & \\
-1 & $x_{1}$ & $2 / 13$ & 1 & 0 & 0 & $1 / 13$ & $1 / 13$ & $-4 / 13$ & \\
\hline
\end{tabular}

Since all rows and column are ignored, hence an optimum solution has been reached. Therefore optimum solution is:

$$
x_{1}=\frac{2}{13}, x_{2}=\frac{4}{13}, x_{3}=\frac{1}{13} . \text { Min. } X=\frac{7}{13} .
$$

Thus, the optimal strategies for player A are:

$$
\begin{gathered}
p_{1}=\frac{x_{1}}{X}=\frac{\frac{2}{13}}{\frac{7}{13}}=\frac{2}{7}, p_{2}=\frac{x_{2}}{X}=\frac{\frac{4}{13}}{\frac{7}{13}}=\frac{4}{7}, \\
p_{3}=\frac{x_{3}}{X}=\frac{1 / 13}{7 / 13}=\frac{1}{7} \\
\text { and }=\frac{1}{X}-k=\frac{13}{7}-2=-\frac{1}{7} .
\end{gathered}
$$

The optimal strategies for player B are obtained from final table of the above problem. This is given by duality rules:

$$
\begin{gathered}
X=Y=\frac{7}{13}, \\
y_{1}=\left[\begin{array}{lll}
-1 & -1 & -1
\end{array}\right]\left[\begin{array}{c}
-6 / 13 \\
2 / 13 \\
1 / 13
\end{array}\right]=\frac{3}{13}, \\
y_{2}=\left[\begin{array}{lll}
-1 & -1 & -1
\end{array}\right]\left[\begin{array}{c}
7 / 13 \\
-11 / 13 \\
1 / 13
\end{array}\right]=\frac{3}{13}, \\
y_{3}=\left[\begin{array}{lll}
-1 & -1 & -1
\end{array}\right]\left[\begin{array}{c}
-2 / 13 \\
5 / 13 \\
-4 / 13
\end{array}\right]=\frac{1}{13} .
\end{gathered}
$$

Hence,

$$
\begin{gathered}
q_{1}=\frac{y_{1}}{Y}=\frac{\frac{3}{13}}{\frac{7}{13}}=\frac{3}{7}, q_{2}=\frac{y_{2}}{Y}=\frac{\frac{3}{13}}{\frac{7}{13}}=\frac{3}{7} \\
q_{3}=\frac{y_{3}}{Y}=\frac{1 / 13}{7 / 13}=\frac{1}{7} \\
\text { and } V=\frac{13}{7}-2=-\frac{1}{7} .
\end{gathered}
$$




\section{CONCLUSIONS}

An alternative method for game theory problems to obtain the solution of linear programming problem has been derived. This technique is useful to apply on numerical problems, reduces the labour work and save valuable time.

\section{REFERENCES}

[1]. G. B. Dantzig: Maximization of linear function of variables subject to linear inequalities, In: 21-Ed. Koopman Cowls Commission Monograph, 13, John Wiley and Sons, Inc., New Yark (1951).

[2]. K. G. Lokhande, N. W. Khobragade, P. G. Khot: Simplex Method: An Alternative Approach, International Journal of Engineering and Innovative Technology, Volume 3, Issue 1, P: 426-428 (2013).

[3]. N. V. Vaidya, N. W. Khobragade: Solution of Game Problems Using New Approach, IJEIT, Vol. 3, Issue 5, 2013.

[4]. N. W. Khobragade and P. G. Khot: Alternative Approach to the Simplex Method-II, Acta Ciencia Indica, Vol.xxx IM, No.3, 651, India (2005).

[5]. S. D. Sharma: Operation Research, Kedar Nath Ram Nath, 132, R. G. Road, Meerut-250001 (U.P.), India.

[6]. S. I. Gass: Linear Programming, 3/e, McGraw-Hill Kogakusha, Tokyo (1969).

[7]. K. P. Ghadle, T. S. Pawar, N. W. Khobragade: Solution of Linear Programming Problem by New Approach, IJEIT, Vol.3, Issue 6. Pp.301-307, 2013

\section{BIOGRAPHIES}

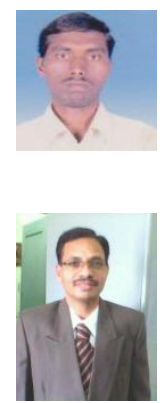

Mr. Tanaji S. Pawar, Research student, Department of mathematics, Dr. Babasaheb Ambedkar Marathwada University, Aurangabad.

Dr. K. P. Ghadle for being M.Sc in Maths he attained Ph.D. He has been teaching since 1996. At present he is working as Associate Professor. Achieved excellent experiences in Research for 15 years in the area of Boundary value problems and its application. Published more than 45 research papers in reputed journals. Four students awarded Ph.D Degree and four students working for award of Ph.D. Degree under their guidance. 